\title{
PRELIMINARY PLANS FOR USE OF HOUGH-POWELL BUBBLE
}

LEGAL NOTICE DENSTTY NEASURENENTS

This report was prepared as an nccount of Government sponsored work. Nelther
States, nor the Commission, nor any person acting on behalf of the Commision:

A. Makes any warranty or representation, expressed or implied, with respect to the accu-

racy, completeness, or usefulness of the information contained in this report, or that infringe
of any information, apparatus, method, or process disclosed in this report may not

privately owned rights; or

B. Assumes any labilites wit method, or process disclosed in this report.

se of any information, apparatus, method, or prehalf of the Commission" includes any em-

As used contractor of the Commission, or employee of such contractor, to the extent that

such employee or contractor of the Commission, or fmployee of such contractor prepares,

with the Commission, or his employment with such contractor.

R. C. Strand

Introduction

In this report we summarize the current plans for the use of bubble density measurements during the early operational stages of the Hough-Powell system. Our attention is focused on measurements of twenty-inch chamber film.

We plan to use the bubble density measurements to eliminate obviously wrong mass assignments. This will save computer time that would otherwise be wasted on kinematic fits and it will reduce the amount of output paper. We also hope to be able to resolve the mass assignment ambiguities that remain after kinematic fits, without taking the events back to the scan table.

We welcome suggestions related to a particular bubble density application or to a good idea that we have overlooked.

This report is divided into four parts which correspond to the four major IBM 7090 programs of the Hough-Powell system. I Except as noted these programs are coded and are being checked. As a heading for each part, the name of the program, the code (FAP or FORTRAN), and the programmer in charge are given. We will take one event through the complete system. Some general conclusions follow the description of the last program. 


\section{DISCLAIMER}

This report was prepared as an account of work sponsored by an agency of the United States Government. Neither the United States Government nor any agency Thereof, nor any of their employees, makes any warranty, express or implied, or assumes any legal liability or responsibility for the accuracy, completeness, or usefulness of any information, apparatus, product, or process disclosed, or represents that its use would not infringe privately owned rights. Reference herein to any specific commercial product, process, or service by trade name, trademark, manufacturer, or otherwise does not necessarily constitute or imply its endorsement, recommendation, or favoring by the United States Government or any agency thereof. The views and opinions of authors expressed herein do not necessarily state or reflect those of the United States Government or any agency thereof. 


\section{DISCLAIMER}

Portions of this document may be illegible in electronic image products. Images are produced from the best available original document. 
I. HAZE (FAP): Neil Webre

HAZE is the first major program of the system. It runs on line with the Hough-Powell flying spot digitizer on which is mounted one roll of film.

Previously at a special scan table, "human guidance" is prepared for HAZE. Three views of the event are scanned and necessary information is punched on cards by special hardware at the scan table. First the usual indicative information for the event is punched. Then, in each of three views, ? fiducial marks are digitized and three points are digitized on each track. These measurements are said to be "rough digitized" in contrast with the precise flying spot measurements.

HAZE accepts input from the scan table that telis it which frame contains the event. HAZE causes the film to be advanced to this frame. According to the average projected angle of the rough digitized track, HAZE selects one of the two orthogonal flying spot scan modes for the precise measurement. The coordinates of every bubble in the frame are transmitted to HAZE through an IBM Direct Data Connection. Among these coordinates HAZE finds the precise fiducials. The points on a track are located within a "road". determined by the three rough digitizations. The points outside of the road are forgotten by HAZE. All of the points in the road are "gated".into groups of twenty. In each group the points which belong to the track are located in the highest bin of a histogram taken transverse to the road. These are called the filtered points and they are averaged to give one "master" point to be used for 
stereo reconstruction of the track.

The spacings of the filtered points contain the bubble density information. The maximum likelihood bubble

density ${ }^{2}$ is computed in the next program from the total number of gaps, the total number of scan lines, and the total number of digitizations associated with the track. For each track HAZE will collect this information and transmit it to the next program. HAZE must also transmit. which one of the two orthogonal scan modes it. used to measure the track.

We see that HAZE output resembles the data that is usually obtained from a measuring projector.

The performance of HAZE will be monitored periodically to make sure that the filtered points are the complete set of points associated with the track. To accomplish this, HAZE will produce a special output tape of the gated points and the filtered points. These points will be examined by a separate program. 
II. FOG 135 (FAP): Phil Connolly

The basic geometry program, FOG 135, accepts as input the binary output of HAZE. For each track, FOG 135 selects the two best oriented views from which it makes the stereo reconstruction.

For each view of a track FOG 135 will compinte the measured bubble density on the film and its error. This computation will include an adjustment for the average angle between the scan lines and the track. The film bubble density in each view will be transmitted through to the last program for listing. These are the bubble densities as the eye should see. them.

Geometrical adjustments must be applied to the film bubble density in order to obtain the true bubble density of the track. The largest adjustment comes from the angle between the track at its midpoint and a line to the center of the camera lens. This adjustment includes the effect of dip angle, mean projected angle, and mean $x-y$ position in the chamber. A smaller adjustment must be made for the chamber to film magnification at the mean depth of the track. In our use of bubble density we assume that the uncertainties in position and angle are negligible. Hence, the true bubble density error is determined by the statistical error of the film bubble density; the momentum error is determined by the curvature error. A considerable simplification results from uncoupling the momentum and bubble density measurements. 
FOG 135 must select for further use the true bubble density measurement which has the smallest error. In general the camera which sees the track most nearly head-on will yield the smallest statistical error because the maximum number of gaps are visible in this configuration.

For each track FOG 135 output contains measurements of momentum, angles, and the true bubble density. The output will aiso contain three measurements of the film bubble density and three measurements of the true bubble density for each track. One measurement was obtained from each view. As was mentioned, the film bubble densities will be compared with the pictures. The three individual true bubble density measurements will be useful to check the bubble density measurement process.

Coding of the bubble density computations in FOG 135 will begin soon. 


\section{CLOUDY (FAP): Phil Connolly}

The kinematic fitting program, CLOUDY, accepts binary input information from FOG 135.

First the bubble density measurements will be used by CLOUDY to prevent useless kinematic fits to mass assignments which are in obvious disagreement with the momenta and bubble density measurements. 3

CLOUDY prepares a set of masses which are consistent with the known conservation laws. For each track the mass-dependent, mid-point momentum and error are computed from stereo information. The mass set is next tested by CLOUDY to see if it is consistent with the measured momenta and bubble densities. To understand how this is done we recall that the bubble density, $D$, and the velocity, $\beta$, of a particle are related by the equation (I)

$$
D=A \cdot B^{-2}
$$

where $A$ is a constant for fixed bubble chamber conditions. 4 The mass of the particle enters equation (1.) through the relationship between velocity, momentum, $P$, and mass, $M$, shown in equation (2).

$$
\beta^{2}=P^{2} /\left(M^{2}+P^{2}\right)
$$

CLOUDY makes a least squares fit for the mass set, the measured momenta, and the measured true bubble density. ${ }^{5}$ The fit is constrained according to a set of equations of the type (I). There is one constraint equation for each track. Since the constant, A, of equation (I) is treated as an unknown, the number of degrees of 
freedom is one less than the number of tracks. We emphasize that angles and kinematics are left out of this fit The chi-squared. value for this fit is tested to see if the proposed mass set is reasonable.

If the mass set is accepted, CLOUDY performs the standard kinematic fit and the process is repeated until all of the nossible mass assignmënts have been considered.

CLOUDY output contains information collected from HAZE and FOG 135. It contains the results of each bubble density fit to the measured.momenta and the results of each kinematic fit.

At this point we hope to have eliminated those mass assionments that could have been eliminated by eye. We are motivated by the potential reduction of computing time and paper work. Those mass assignment ambiguities which remain after kinematic fits will be considered in the next program. 
IV. The Suffolk County FAIR (FORTRAN): Dave Stonehill

The final program, FAIR, accepts binary input from CLOUDY, performs calculations on these data, and prepares lists of the useful' results. We will not describe all of the many featuresof the Suffolk County FAIR. For this information the reader is referred to Dave Stonehill's report. 6

We will discuss the further use of bubble density measurements to resolve any mass assignment ambiguities that remain after the kinematic fits have been made.

A subroutine in FAIR will perform a bubble density fit to the fitted momenta from a particular kinematic fit. The resultant chi-squared value can be used to select the best mass assignment(s). We note that the fitted momenta are in general changed from their measured values and the fitted errors are smaller than the errors of measurement. Thus, there is hope that the second bubble density fit will favor one mass assignment. This step is now performed by physicists who take the kinematic results back to the scan table for a final judgement. 
V. Conclusions

We have described a use for bubble density measurements. that involves three steps: a pre-kinematics bubble density fit, the standard kinematic fit, and a rost-kinematics bubble density fit. At each step we have a chance to observe the behavior of the program. Mistakes can be isolated at each step. It is true that these three steps could be incorporated into a combined least square fit according to bubble density and kinematics. In the absence of mistakes, this overall fit might provide a better resolution of mass assignment ambiguities.

However, our immediate problem is to determine the range of track configurations, masses, and momenta for which reliable bubble. density measurements can be obtained. Visual bubble densities will provide a good semi-quantitative calibration. The tedious direct bubble density measurements on measuring projectors can be employed. It has been suggested that special bubble chamber pictures in the mass-separated beam would provide a good calibration. The four constraint kinematics of elastic scattering events might be used to calibrate bubble densities. As yet no firm plans have been made to use a particular calibrator.

Many people who are working on the Hough-Powell system have contributed to the plans presented in this report. We encourage comments and suggestions on our plans. 


\section{References}

1. H. S. White, "FOG, CLOUDY, and FAIR Program's", UCRL-9476, p. 12,1960 .

2. R.C. Strand, "Bubble Density Measurement with the Hough-Powell Digitizer", BNL Bubble.Chamber Report G-34, January 1.963.

3. A.M. Thorndike, "An Elementary Use for Bubble Density", June 14,1962 .

4.W. J. Willis, W. Fowler, and D.C. Rahm, Phys. Rev. 108, 1046 (1957); V.P. Kenney, Phys. Rev. I19, 432 (1960); R. Lea. and C. Vittitoe, "Bubble Density Measurements for Particle Identification II", BNL Bubble Chamber Report D-69II, 7/24/61.

5. The program for a bubble density fit to momenta and a mass set has been coded and tested by Joan Franz. Fits have been obtained. A forthcoming report will describe this program. 6. D. Stonehill, "The Suffolk County Fair Preliminary Report", BNL Bubble Chamber Group Report F-93; D. L. Stonehill, "The Suffolk County Fair System", BNL Bubble Chamber Group Report F-107. 